\title{
Heterogeneous Architecture for Ad hoc Networks
}

\author{
Xue-bing Wang \\ Department of Information and Computer Science \\ Sheng Li College, China University of Petroleum \\ Dongying Shandong, China \\ bbxxww2002@163.com
}

\begin{abstract}
By making small adjustment to general ad hoc network architecture, we build a network topology with short average path length and high clustering coefficient, which are two important metrics of ad hoc networks. Furthermore, an efficient probabilistic flooding routing algorithm is proposed based on this network model. Simulation results show that this architecture behaves better performance than its ordinary counterpart.
\end{abstract}

Keywords- ad hoc networks; average path length; clustering coefficient; probabilistic flooding

\section{INTRODUCTION}

Ad hoc networks are formed by a group of nodes which are all equipped with wireless transceiver and communicate with each other over a wireless channel. They operate in a decentralized and self-organizing manner and do not rely on network infrastructure. Each node acts as a router to forward traffic toward its destination. Typical ad hoc network architecture is homogeneous. It is to say that all nodes have a same communication range and are on the equal status, so there is no one to be more important than others.

There are a lot of metrics of ad hoc networks, such as coverage, connectivity, mobility, etc. Average path length and clustering coefficient are also two important metrics of ad hoc networks. In a network, the distance $d_{i j}$ between node $i$ and node $j$ is defined to be the number of edges on the shortest path between $i$ and $j$. Average path length, also being called characteristic path length, is defined to be the arithmetically average value of $d_{i j}$ for each pair of nodes, i.e.

$$
L=\frac{1}{\frac{1}{2} N(N+1)} \sum_{i \geq j} d_{i j}
$$

$N$ represents the number of nodes in the network. Average path length depicts the network scale, and decides the end-to-end delay of traffic packet.

Clustering coefficient of a node $i$ is defined to be the number of edges among its neighbors divided by the possible maximum number of edges between them, i.e.

$$
C_{i}=2 E_{i} /\left(k_{i}\left(k_{i}-1\right)\right)
$$

$E_{i}$ is the number of existent edges between neighbors of node $i . k_{i}$ represents the number of neighbors. Clustering coefficient $C$ of the whole network is defined to be the average value of $C_{i}$. It is certain that $C$ should satisfy $0 \leqq C \leqq 1$. Clustering coefficient depicts clustering effect of nodes in network. A complete random graph, such as E-R random graph [1], has clustering coefficient $\mathrm{C}=\mathrm{O}\left(\mathrm{N}^{-1}\right)$, but an actual network will have a much higher one.

A homogeneous ad hoc network will have a long average path length and a low clustering coefficient. Both will result in a large number of hop counts, long end-to-end delay, and a great deal of power consumption, etc. But if we make some modification to the communication range of some transceivers, we will get obvious benefit. Because the communication range is not identical in this network model, we call it heterogeneous architecture. Correspondingly, the typical ad hoc network architecture is called homogeneous.

In this paper, we also design an uneven probabilistic flooding algorithm based on the heterogeneous network architecture. This algorithm not only can be used as an independent message dissemination technique, but also can act as an assistant tool for route discovery and route maintenance of other routing algorithm. Our algorithm applies the idea of probabilistic flooding to reduce the number of transmission so as to decrease the channel contention and collision. But the transmission probability is not assigned uniformly among all nodes so it is an uneven probabilistic flooding algorithm. Simulation shows that such a flooding algorithm combined with the heterogeneous networks will produce much higher efficiency than a usual one.

The rest of this paper is organized as follows. In section II we will build a heterogeneous ad hoc model. In section III, we propose an uneven probabilistic flooding algorithm which is based on this model. Simulation experiments are done to compare the performance difference between homogeneous networks and heterogeneous ones. Section IV gives the conclusion.

\section{Heterogeneous Network ArChitecture}

In a typical ad hoc network, generally speaking, every node is equipped with a pair of transceiver. All nodes have the same power supply, which translates into the same communication range. Such homogeneous network architecture can be modeled as random geometric graph [2]. Nodes in such a model can communicate with each other only if the distance between them is less than communication range, which is decided by power. If the distance exceeds the range limit, nodes cannot see each other.

In the heterogeneous model, some nodes are selected randomly and equipped with transmitter of two times communication distance of other nodes. These nodes are called special nodes to be distinguished from the ordinary 
ones. Simulation shows that only if a small part of nodes are selected as special ones, the parameters of average path length and clustering coefficient will improved greatly.

Simulation setting: 400 nodes are arranged on the plane to form an array of 20 rows and 20 columns (see fig.1). The communication range of an ordinary node is set to guarantee that it has 4 neighbors at most. The communication range of a special node is set to be two times of an ordinary one, so it will have 8 neighbors. At the beginning, there are no special nodes, so it forms a homogeneous network (see fig. 1 a). Then the number of special nodes is added little by little (fig. 1 b) until all nodes become special (fig.1 c). It should be noted that when all nodes become special, the network becomes a homogeneous one again. For each scenario, the average path length and clustering coefficient are collected and illustrated in fig. 2 and fig. 3. It is interesting that when the proportion of special nodes is changed from 0 to 20 percent, there is an obvious improvement for the statistics. From then on, further increase of special nodes will not bring abrupt benefit. By dint of physical terminology, such phenomenon is called phase transition [3]. The phenomenon of phase transition shows that only 20 percent of nodes are needed to be special to decrease average path length and increase clustering coefficient greatly. Although the experiment is done on a grid network, the same conclusion can also be drawn with a random topology.

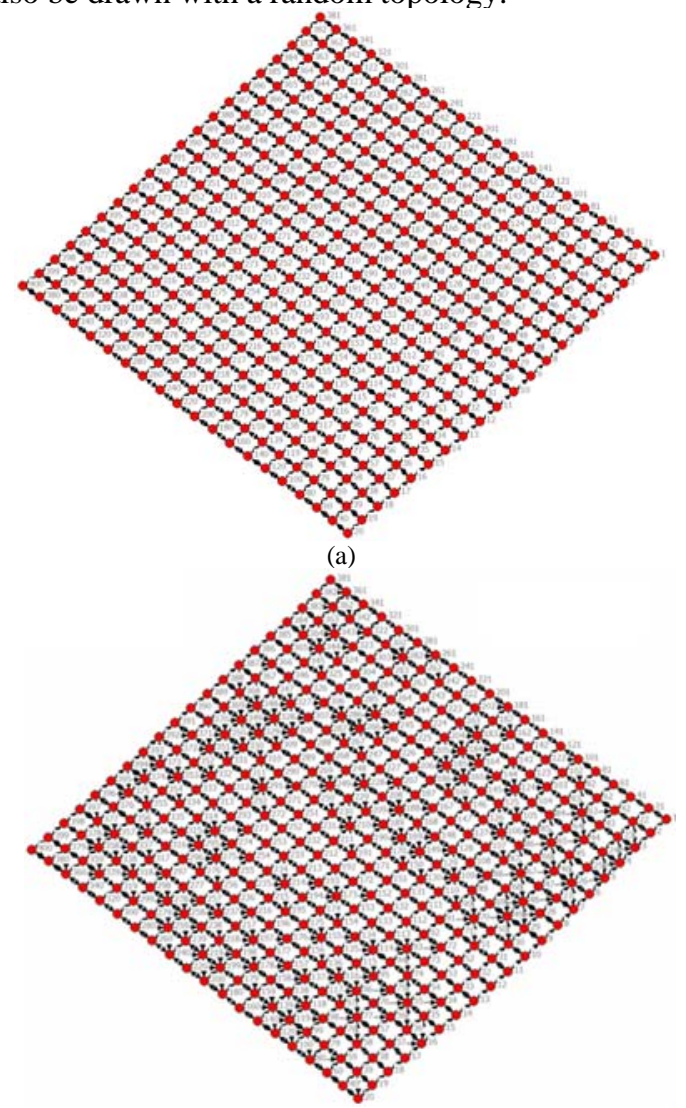

(b)

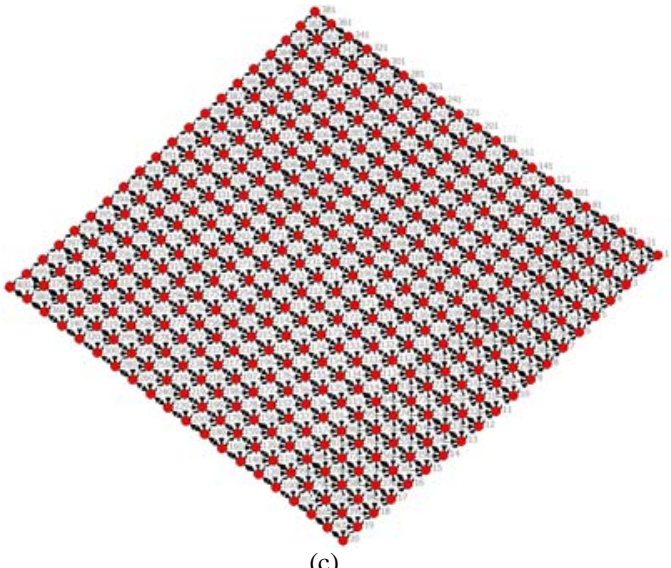

Figure 1. (a) no special nodes (b) 80 special nodes (c) 400 special nodes

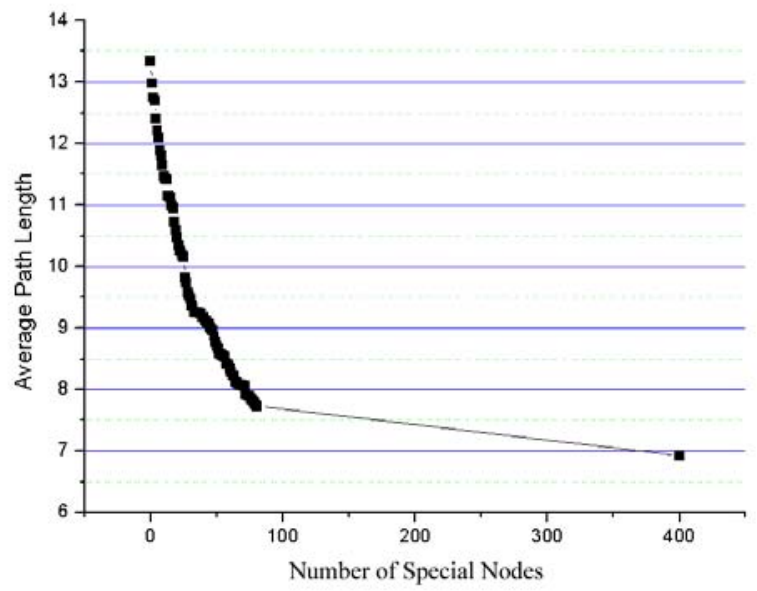

Figure 2. Average path length of heterogeneous network model

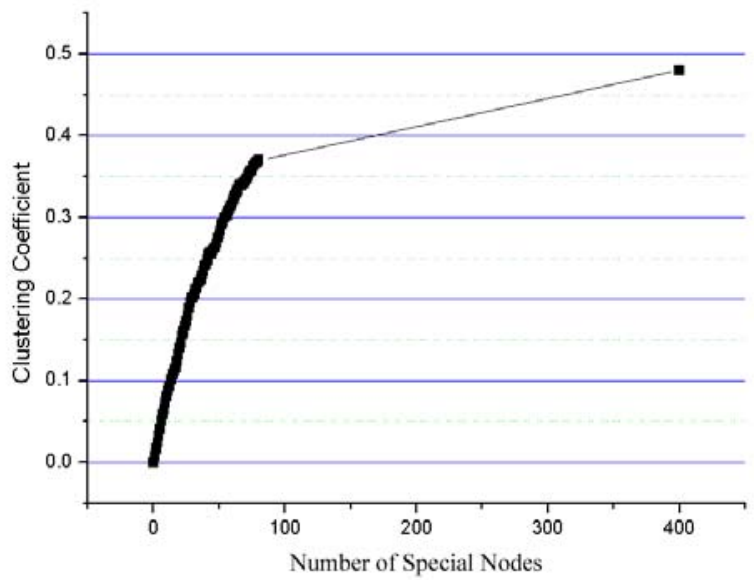

Figure 3. Clustering coefficient of heterogeneous network model

\section{UneVEn Probabilistic FloOding Algorithm}

Probabilistic flooding algorithm was advanced by Sasson [4] originally. It is proved to be an efficient message dissemination technique for ad hoc networks. Traditional flooding algorithm demands mobile to broadcast message as 
soon as it is received. Such way will produce a large amount of packet collision. In probabilistic flooding strategy, node will broadcast the received message with probability $0<p<1$. Correspondingly, traditional flooding method uses the probability 1 . It is proved, with the theory of percolation, that when $p$ is increased, the packet delivery ratio will undergo abrupt transition from 0 to 1 . It is to say that there is a certain bound $p_{c}$, and the flooding probability over $p_{c}$ will not provide any significant improvement.

In this section, we design a novel probabilistic flooding algorithm based on the heterogeneous network model. In this model, we equip every node with one transmitter and two receivers. There are two transmission frequency bands available in the overall network, one for special nodes' transmitter and the other for ordinary ones. All nodes can receive and process the signal of the two frequency bands. The power of special nodes' transmitter is set to make its communication distance to be two times of the ordinary ones. Additionally, we endow special nodes with bigger transmission probability than ordinary ones, so it is called uneven probabilistic flooding algorithm. To compare the performance of the uneven probabilistic flooding algorithm with a general probability flooding algorithm, we perform two simulation-based experiments in the network simulation environment OPNET.

Simulation setting for ordinary probabilistic flooding algorithm based on homogeneous network: 400 nodes are randomly distributed on the plane with size of $2000 \mathrm{~m} * 2000 \mathrm{~m}$ (see fig. 4). The communication radius of nodes is set to be $200 \mathrm{~m}$. A node near the domain center $(1000,1000)$ is selected as broadcast source and other nodes act as destinations. In simulation, with the increase of transmission probability from 0 to 1 , two target statistics are collected. The first is the number of nodes received packets, which reflects the coverage area. The second statistics is hop count, which correlates with average path length.

Simulation setting for uneven probabilistic flooding algorithm based on heterogeneous network model is similar to previous experiment, except that the communication range of a special node is set to be $300 \mathrm{~m}$, and it is $150 \mathrm{~m}$ for general nodes. There are totally 80 (20 percent) nodes to be special. Simulation results for the two experiments are compared in fig. 6 and fig. 7. We can draw the conclusion that the uneven probabilistic flooding algorithm combined with heterogeneous network model outperforms the ordinary probabilistic flooding based on homogeneous greatly. Firstly, the former get 90 percent broadcast coverage with transmission probability 0.2 , but the latter need the probability more than 0.6 to achieve this. Secondly, hop count in the former model is far less than the latter, which means short transmission delay. Last but not the least, in heterogeneous model, there are 320 nodes whose communication radius is $150 \mathrm{~m}$ and 80 nodes whose radius is $300 \mathrm{~m}$. But all nodes in homogeneous model will use $200 \mathrm{~m}$ communication distance. It can be calculated, by the formula of transmission in free space, the total power consumption of the former is about $13.75 \%$ less than the latter.

\section{CONCLUSION}

The heterogeneous network model gets obvious benefit, mainly in average path length and clustering coefficient, at a cost of more power consumption for only 20 percent nodes. The uneven probabilistic flooding algorithm based on this model is proved to be more efficient than the traditional one. Moreover, this novel algorithm is very simple. It is easy for implementation without any cost of control. This algorithm has the advantage of less broadcast collision, short transmission delay, and less power consumption. But it should be noted that although the total power consumption of the uneven probabilistic flooding algorithm is less than the traditional one, it is much higher for special nodes. This demands special power supply for these special nodes.
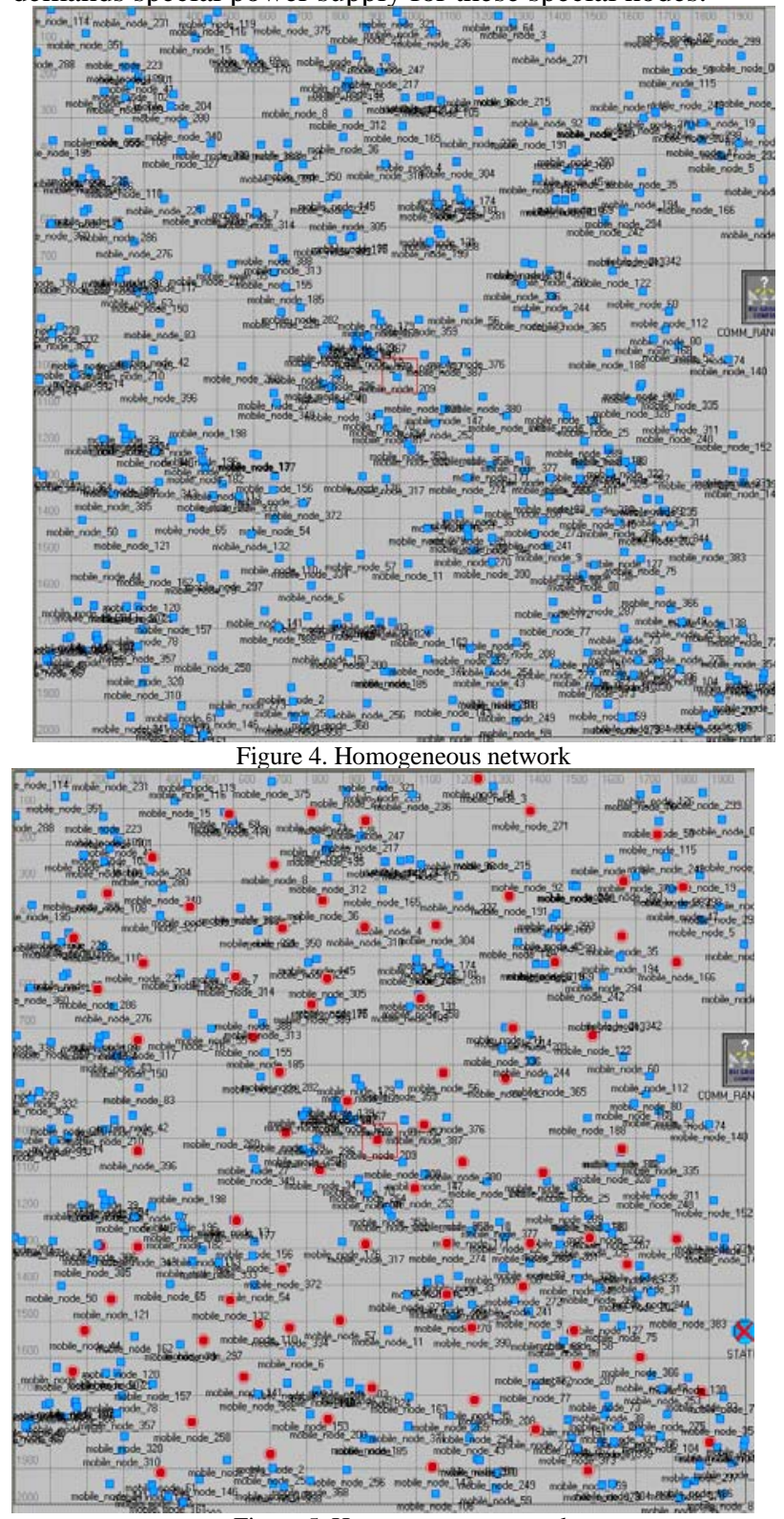

Figure 5. Heterogeneous network 


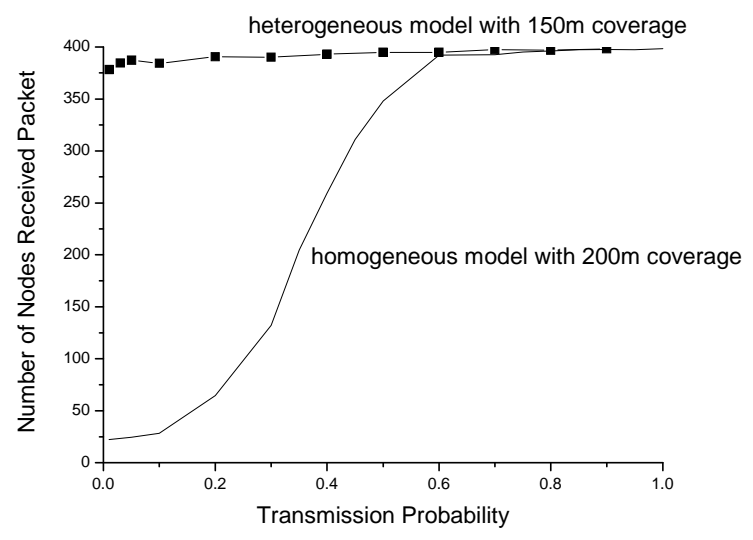

Figure 6. Comparison of packet delivery ratio

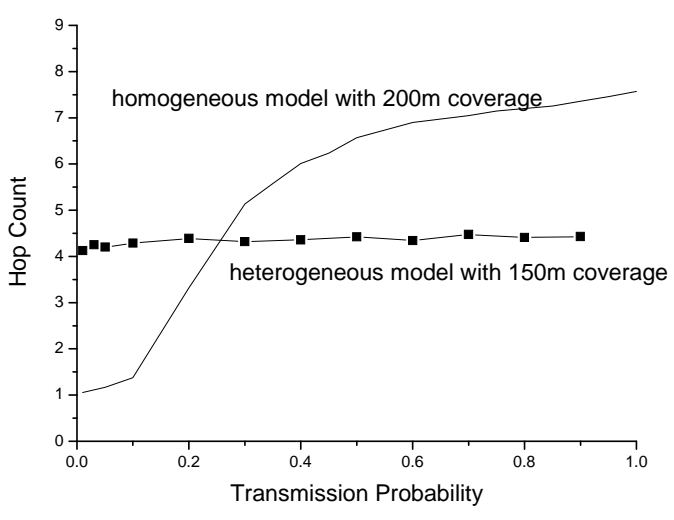

Figure 7. Comparison of hop count

\section{REFERENCES}

[1] B.Bollobas, Random graphs, Cambridge University Press, 2001

[2] M.Penrose. Random geometric graphs. Oxford university press, 2003

[3] Bhaskar Krishnamachari, Stephen B. Wicker, Ramon Bejar. Phase transition phenomena in wireless Ad hoc networks. Global Telecommunications Conference, 2001. Globecom'01 IEEE Vol5, pp.2921 - 2925 .

[4] Yoav Sasson, David Cavin, Andre Schiper. Probabilistic broadcast for flooding in wireless mobile Ad hoc networks. 2003 IEEE Wireless Communications and Networking Conference Record (Cat. No. 03 TH8659), 2003, vol.2, pp. 1124-1130 